\title{
THE FIXED POINT PROPERTY FOR CONTINUA APPROXIMATED FROM WITHIN BY PEANO CONTINUA WITH THIS PROPERTY
}

\author{
AKIRA TOMINAGA
}

\begin{abstract}
Let $X$ be a continuum that is approximated from within by Peano subcontinua with the fixed point property (FPP). Then we show a sufficient condition that $X$ has FPP. As a consequence we have that the Cartesian product of $n$ Warsaw circles is a $T^{n}$-like continuum with FPP and the $n$-fold suspension of Warsaw circle is an $S^{n}$-like continuum with FPP.
\end{abstract}

1. Introduction. Let $X$ be a space that is approximated from within by subsets with the fixed point property (abbreviated FPP), i.e., containing a monotone increasing sequence $C_{1} \subset C_{2} \subset \cdots$ of subsets with FPP such that $X=\bigcup_{i} C_{i}$. Then it is natural to ask when $X$ has FPP. G. S. Young [7] proved that every arcwise connected Hausdorff space in which every monotone collection of arcs is contained in an arc has FPP. (Subsequently he mentioned that in this result compactness is not required [8].) L. E. Ward, Jr. [5] generalized both Young's theorem above and Borsuk's theorem [2] that an arcwise connected hereditarily unicoherent metric curve has FPP.

In this paper we show that if $X$ is approximated from within by Peano subcontinua with FPP and satisfies certain conditions, then it has FPP. As a consequence we have that for every positive integer $n$ the Cartesian product of $n$ Warsaw circles is a $T^{n}$-like continuum with FPP and the $n$-fold suspension of Warsaw circle is an $S^{n}$-like continuum with FPP, where $T^{n}$ and $S^{n}$ mean an $n$-dimensional torus and an $n$-sphere, respectively. Here we refer to the fact that E. Dyer [4] proved that the Cartesian product of $n$ chainable continua has FPP.

\section{Main theorem.}

DEFinition. A continuum is a compact connected metric space and a Peano continuum is a locally connected continuum. A map is a continuous function. Let $(M, d)$ be a metric space and $\varepsilon$ a positive number. A map $f: M \rightarrow M$ is said to be $\varepsilon$-near to the identity map or simply to be $\varepsilon$-near if $d(x, f(x))<\varepsilon$ for every $x \in M$.

THEOREM 1. Let $X$ be a continuum for which there exists a sequence $C_{1} \subset$ $C_{2} \subset \cdots$ of Peano continua such that $X=\bigcup_{i} C_{i}$ and every $C_{i}$ has FPP. If the following two statements hold, then $X$ has FPP.

(1) For every $\varepsilon>0$, there exists a $C_{i}$ and a function $f: X \rightarrow X$ such that for each $s>i$ the restriction $f \mid C_{s}$ is an $\varepsilon$-near map of $C_{s}$ to $C_{i}$.

Received by the editors May 23, 1983 and, in revised form, August 19, 1983. A summary of the contents of this paper and the other several results about FPP by the author appeared in RIMS Kokyuroku, no. 509 (January, 1984), 41-47, Research Institute for Mathematical Sciences, Kyoto University, Kyoto, Japan.

1980 Mathematics Subject Classification. Primary 54F20, 54H25.

Key words and phrases. Fixed point property, continuum, Warsaw circle. 
(2) There exists a closed subset $A$, which may be empty, of $\bigcap_{j} \overline{X-C_{j}}$, such that every $f$ in (1) is continuous on $A, f(A) \subset C_{1}$ and every point $x \in \bigcap_{j} \overline{X-C_{j}}-A$ has a neighborhood $U$ whose component containing $x$ lies in $a C_{l}$.

Proof. Let $g: X \rightarrow X$ be any map.

(i) For every $C_{i}$ and every $\delta>0$, there exists a $C_{s}(s>i)$ with $g\left(C_{i}\right)-N_{\delta}(A) \subset$ $C_{s}$, where $N_{\delta}(A)$ is a $\delta$-neighborhood of $A$ in $X$. For otherwise, there are a $C_{i}$ and a positive number $\delta$ such that for every $s>i,\left\{g\left(C_{i}\right)-N_{\delta}(A)\right\} \cap\left(X-C_{s}\right)$ contains a point $x_{s}$. By compactness of $g\left(C_{i}\right)-N_{\delta}(A)$, and by taking a convergent subsequence if necessary, we may assume that $\lim x_{s}=x$ for a point $x$ belonging to both $g\left(C_{i}\right)-N_{\delta}(A)$ and $\bigcap_{s} \overline{X-C_{s}}$. Then we can find $U$ and $C_{l}$ satisfying condition (2). Since $C_{i}$ is a Peano continuum, so is $g\left(C_{i}\right)$ by continuity of $g$. Hence there exists a neighborhood $V$ of $x$ in $X$ such that $g\left(C_{i}\right) \cap V$ is contained in the component $K$ of $U$ with $x \in K$. Since $\left\{x_{s}\right\}$ converges to $x$, a point $x_{s}(s>l)$ lies in both $\left\{g\left(C_{i}\right)-N_{\delta}(A)\right\} \cap V$ and $X-C_{s}$. Since $\left\{g\left(C_{i}\right)-N_{\delta}(A)\right\} \cap V \subset K$ and $X-C_{s} \subset X-C_{l}$, it follows that $K \cap\left(X-C_{l}\right) \neq \emptyset$, which contradicts (2). Hence the assertion (i) holds.

(ii) For every $C_{i}, f g\left(C_{i}\right) \subset C_{i}$ holds, where $f$ is the function in (1). For, using (i) and noting that $f$ carries $C_{s}$ into $C_{i}$, we have $f\left(g\left(C_{i}\right)-N_{\delta}(A)\right) \subset C_{i}$ for every $\delta>0$. Since $A$ is closed, we have $\bigcap_{\delta>0} N_{\delta}(A)=A$. Hence

$$
C_{i} \supset \bigcup_{\delta>0} f\left(g\left(C_{i}\right)-N_{\delta}(A)\right)=f\left(g\left(C_{i}\right)-\bigcap_{\delta>0} N_{\delta}(A)\right)=f\left(g\left(C_{i}\right)-A\right) .
$$

Since $f(A) \subset C_{1}$ by our assumption and $C_{1} \subset C_{i}$, we get $f g\left(C_{i}\right) \subset C_{i}$.

(iii) The map $g$ leaves a point of $X$ fixed. To show this, we must show that the function $f g$ is continuous on $C_{i}$. By (i) $g\left(C_{i}\right)-N_{\delta}(A) \subset C_{s}$ holds for every $\delta>0$ and every $s>i$. Since $f$ is continuous on $C_{s}$ by $(1)$, so is $f$ on $g\left(C_{i}\right)-N_{\delta}(A)$, and so is $f$ on $g\left(C_{i}\right)-A$. By (2) $f$ is continuous on $A$. Thus $f$ is continuous on $g\left(C_{i}\right)$.

Now let $\varepsilon$ be any positive number, and $C_{i}$ a set as in (1). Since $C_{i}$ has FPP, there exists a point $y \in C_{i}$ with $f g(y)=y$. Thus we have $d(y, g(y))=d(f g(y), g(y))<\varepsilon$, because $f$ is $\varepsilon$-near. Since $\varepsilon$ is arbitrary and $X$ is compact, $g$ leaves a point of $X$ fixed.

REMARK 1. An $n$-sphere $S^{n}(n \geq 1)$ satisfies condition (1) in Theorem 1 but has no FPP.

REMARK 2. In the above theorem, if a point $x \in X$ is not in $\bigcap_{j} \overline{X-C_{j}}$, then $X$ is locally connected at $x$. However, if $x$ is in $\bigcap_{j} \overline{X-C_{j}}-A$, then $X$ is not locally connected at $x$.

3. Cartesian products. It is well known that even if both $X_{1}$ and $X_{2}$ have FPP, the Cartesian product $X_{1} \times X_{2}$ does not necessarily have FPP (cf. [3]). For continua such as in Theorem 1 , we have

THEOREM 2. Suppose that for each $k(1 \leq k \leq n), X_{k}$ and $C_{k 1} \subset C_{k 2} \subset \cdots$ satisfy the conditions in Theorem 1. If every $C_{1 i} \times C_{2 i} \times \cdots \times C_{n i}(i=1,2, \ldots)$ has FPP, then so does $X_{1} \times X_{2} \times \cdots \times X_{n}$.

PROOF. It is sufficient to show that $X=X_{1} \times \cdots \times X_{n}$ and $C_{i}=C_{1 i} \times \cdots \times C_{n i}$ $(i=1,2, \ldots)$ satisfy the conditions in Theorem 1 . Obviously $C_{i}$ are Peano continua in the continuum $X$ such that $X=\bigcup_{i} C_{i}$. Now let $k$ be any integer in $\{1,2, \ldots, n\}$. 
Condition (1). For every $\varepsilon>0$, there exist a $C_{k i}$ and a function $f_{k}: X_{k} \rightarrow X_{k}$ such that for each $s>i$ the restriction $f_{k} \mid C_{k s}$ is an $\varepsilon / \sqrt{n}$-near map of $C_{k s}$ to $C_{k i}$. Let $f$ be the function of $X$ to itself defined by $f(x)=\left(f_{1}\left(x_{1}\right), f_{2}\left(x_{2}\right), \ldots, f_{n}\left(x_{n}\right)\right)$ for $x=\left(x_{1}, x_{2}, \ldots, x_{n}\right)$. Then the restriction $f \mid C_{s}$ is an $\varepsilon$-near map of $C_{s}$ to $C_{i}$ under the metric $\left(d_{1}^{2}+\cdots+d_{n}^{2}\right)^{1 / 2}$ on $X$, where $d_{k}$ is a metric on $X_{k}$.

Condition (2). By a simple argument we have the equality $\bigcap_{j} \overline{X-C_{j}}=\bigcup_{k} X_{1} \times$ $\cdots \times X_{k-1} \times L_{k} \times X_{k+1} \times \cdots \times X_{n}$, where $L_{k}=\bigcap_{j} \overline{X_{k}-C_{k j}}$. Now by (2) in Theorem 1 there exists a closed subset $A_{k}$ of $L_{k}$ such that $f_{k}\left(A_{k}\right) \subset C_{k 1}$ and every $y_{k} \in L_{k}-A_{k}$ has a neighborhood $U_{k}$ whose component containing $y_{k}$ lies in a $C_{k l}$. Denote $A_{1} \times \cdots \times A_{n}$ by $A$. Since $A_{k}(1 \leq k \leq n)$ is compact, $A$ is closed in $\bigcap_{j} \overline{X-C_{j}}$. Moreover, $f$ is continuous on $A$ and $f(A)=f_{1}\left(A_{1}\right) \times \cdots \times f_{n}\left(A_{n}\right) \subset$ $C_{11} \times \cdots \times C_{n 1}=C_{1}$.

Let $x=\left(x_{1}, \ldots, x_{n}\right)$ be a point in $\bigcap_{j} \overline{X-C_{j}}-A$. Then an $x_{k}$ is not in $A_{k}$. If $x_{k} \in X_{k}-L_{k}$, then $x_{k}$ has a connected neighborhood $U_{k}$ in $X_{k}$ contained in a $C_{k l_{k}}$. (See Remark 2 in the previous section.) If $x_{k} \in L_{k}-A_{k}$, then there exists a neighborhood $U_{k}$ of $x_{k}$ in $X_{k}$ whose component containing $x_{k}$ lies in a $C_{k l_{k}}$ for some $l_{k}$. Then $U=U_{1} \times \cdots \times U_{n}$ is a neighborhood of $x$ in $X$ whose component containing $x$ lies in $C_{l}=C_{1 l} \times \cdots \times C_{n l}$, where $l=\max _{k} l_{k}$.

Thus $X$ and $C_{i}$ satisfy the conditions in Theorem 1, and our proof is complete.

Definition. Let $X$ and $Y$ be compact metric spaces. Then $X$ is said to be $Y$-like if for every $\varepsilon>0$ there is a map $f$ of $X$ onto $Y$ such that for every $y \in Y$ the diameter of $f^{-1}(y)$ is less than $\varepsilon$.

COROLlaRY 1. For every positive integer $n$, the Cartesian product of $n$ Warsaw circles is a $T^{n}$-like continuum with FPP.

Proof. Let $X$ be the Warsaw circle (the $\sin (1 / x)$ circle). There is a monotone increasing sequence of $\operatorname{arcs} C_{i}$ such that $X=\bigcup_{i} C_{i}$ and $\bigcap_{j} \overline{X-C_{j}}$ is the limiting arc. Then $X, C_{i}$ satisfy the conditions of Theorem 1 , in which $A=\emptyset$. Note that if $P_{i}$ is $Q_{i}$-like $(i=1,2)$, then $P_{1} \times P_{2}$ is $\left(Q_{1} \times Q_{2}\right)$-like. Hence by Theorem 2 , the Cartesian product of $n$ Warsaw circles is a $T^{n}$-like continuum with FPP.

REMARK 3. The Cartesian product $X=X_{1} \times X_{2} \times \cdots$ of a countable number of Warsaw circles $X_{i}$ is a $T^{\omega}$-like continuum with FPP. For let $\left(x_{1}, x_{2}, \ldots\right)$ be a constant point of $X$, and define a map $f_{i}: X \rightarrow X_{1} \times \cdots \times X_{i} \times\left(x_{i+1}\right) \times \cdots$ by $f_{i}\left(y_{1}, \ldots, y_{i}, y_{i+1}, \ldots\right)=\left(y_{1}, \ldots, y_{i}, x_{i+1}, \ldots\right)$. Then for every $\varepsilon>0$ there exists an $f_{i}$ which is an $\varepsilon$-near map of $X$ to $X_{1} \times \cdots \times X_{i} \times\left(x_{i+1}\right) \times \cdots$ with FPP. Hence $X$ has FPP.

REMARK 4. The Cartesian product of the above $T^{n}$-like continuum and an $m$ cell $(1 \leq m \leq \omega)$ has FPP, because every $m$-cell satisfies the conditions of Theorem 1.

\section{Cones and suspensions.}

DEFINITION. The cone $Z^{\#}$ over a topological space $Z$ is the quotient space of $Z \times[0,1]$ in which $Z \times 0$ is identified to one point $a$, the vertex of $Z^{\#}$. The suspension $Z^{*}$ of $Z$ is the quotient space of $Z \times[-1,1]$ in which $Z \times(-1)$ is identified to one point $a$ and $Z \times 1$ is identified to another point $b$. The points $a, b$ are called the suspension points of $Z^{*}$. 
There is a continuum with FPP, the cone over which has no FPP (cf. $[\mathbf{1}, \mathrm{p}$. $129]$ ). Recently $\mathrm{T}$. Watanabe treated FPP for cones over some general spaces ( $\S 32$ of [6]). For continua such as in Theorem 1, we have

THEOREM 3. Assume that $X$ and $C_{1} \subset C_{2} \subset \cdots$ satisfy the conditions in Theorem 1. If every $C_{i}^{*}\left(C_{i}^{\#}\right)(i=1,2, \ldots)$ has FPP, then so does the suspension of $X$ (the cone over $X$ ).

We shall prove only the suspension case, as the cone case is similar. The following properties of suspension are readily seen.

Lemma. Let $P, Q$ and $P_{\lambda}$ be subsets of a topological space $X$, and $S$ the set consisting of two suspension points of $X^{*}$. Then the following hold.

(1) $\bigcap_{\lambda} P_{\lambda}^{*}-S=\left(\bigcap_{\lambda} P_{\lambda}\right)^{*}-S$.

(2) $(P-Q)^{*}-S=P^{*}-Q^{*}$.

(3) $\overline{P^{*}}=(\bar{P})^{*}$.

(4) $\overline{P^{*}-Q^{*}}=\overline{P-Q}^{*}$.

PROOF OF THEOREM 3 . Let $a, b$ be the suspension points. A point of $X^{*}$ is expressible as $(x, t) \in X \times[-1,1]$, where $(x,-1)=a,(x, 1)=b$ for any $x \in X$. Note that for a metric $d$ on $X$ there is a metric $d^{*}$ on $X^{*}$ so that $d^{*}((x, t),(y, t)) \leq$ $(1-|t|) d(x, y)$ for every $x, y$ in $X$ and $t \in[-1,1]$.

The sets $C_{i}^{*}$ are Peano continua in the continuum $X^{*}$ such that $X^{*}=\bigcup_{i} C_{i}^{*}$. Therefore, to prove the theorem it is sufficient to show that conditions (1) and (2) in Theorem 1 hold.

Condition (1). Let $\varepsilon$ be any positive number. Then there exist a $C_{i}$ and a function $f: X \rightarrow X$ as in (1) of Theorem 1 . If we define $f^{*}: X^{*} \rightarrow X^{*}$ by $f^{*}(x, t)=$ $(f(x), t)$, then every restriction $f^{*} \mid C_{s}^{*}: C_{s}^{*} \rightarrow C_{i}^{*}$ is $\varepsilon$-near, since $d^{*}((x, t),(y, t)) \leq$ $d(x, y)$.

Condition (2). Let $A$ be as in (2) of Theorem 1. Then $A^{*} \subset\left\{\bigcap_{j} \overline{X-C_{j}}\right\}^{*}=$ $\bigcap_{j} \overline{X^{*}-C_{j}^{*}}$ and $A^{*}$ is closed, by (1) and (2) of the lemma. From $f(A) \subset C_{1}$, it follows that $f^{*}\left(A^{*}\right) \subset C_{1}^{*}$. Also it is easily seen that $f^{*}$ is continuous on $A^{*}$. By the lemma the set $\bigcap_{j} \overline{X^{*}-C_{j}^{*}}-A^{*}$ is equal to $\left(\bigcap_{j} \overline{X-C_{j}}-A\right)^{*}-\{a, b\}$. Let $(x, t)$ be any point of this set. Then $x \in \bigcap_{j} \overline{X-C_{j}}-A$ and $-1<t<1$. Therefore there exists a neighborhood $U$ of $x$ whose component $K$ containing $x$ lies in a $C_{l}$. Then $U^{*}-\{a, b\}$ is a neighborhood of $(x, t)$, and its component containing $(x, t)$ is $K^{*}-\{a, b\}$, which lies in $C_{l}^{*}$. Thus we complete our proof.

COROLLARY 2. For every positive integer $n$, the $n$-fold suspension of a Warsaw circle is an $S^{n}$-like continuum with FPP.

Proof (INDUCTION ON $n$ ). We first note that if $P$ is $Q$-like, then $P^{*}$ is $Q^{*}$ like. Since the Warsaw circle is an $S^{1}$-like continuum with FPP, its suspension $X$ is a $S^{2}$-like continuum with FPP by Theorem 3. Again applying the theorem to $X$, in which $A$ is the set of two suspension points, we have an $S^{3}$-like continuum with FPP. Inductively, the $(n-1)$-fold suspension of the Warsaw circle is a desired continuum.

REMARK 5. The Cartesian product of an $m$-cell $(1 \leq m \leq \omega)$ and the above $S^{n}$-like continuum has FPP. 


\section{REFERENCES}

1. R. H. Bing, The elusive fixed point property, Amer. Math. Monthly 76 (1969), 119-132.

2. K. Borsuk, $A$ theorem on fixed points, Bull. Acad. Polon. Sci. Cl. III 2 (1954), 17-20.

3. R. F. Brown, The fixed point property and Cartesian products, Amer. Math. Monthly 89 (1982), 654-668, 677-678.

4. E. Dyer, A fixed point theorem, Proc. Amer. Math. Soc. 7 (1956), 662-672.

5. L. E. Ward, Jr., A fixed point theorem for chained spaces, Pacific J. Math. 9 (1959), 1273-1278.

6. T. Watanabe, Approximative shape theory, preprint, 1982.

7. G. S. Young, The introduction of local connectivity by change of topology, Amer. J. Math. 68 (1946), 479-494.

8. _ Fixed-point theorems for arcurse connected continua, Proc. Amer. Math. Soc. 11 (1960), 880-884.

FACUlty of Integrated ARTS AND SCIEnCES, Hiroshima University, HiRoshima 730, JAPAN 